\title{
Intracytoplasmic Sperm Injection - Factors Affecting Fertilization
}

\author{
Murid Javed and Essam Michael \\ Additional information is available at the end of the chapter
}

http://dx.doi.org/10.5772/50036

\section{Introduction}

The ICSI has become method of choice to achieve fertilization. Fertilization is possible in cases in which the sperm motility and ability to penetrate the zona pellucida are impaired. Injection is possible with sperm obtained from ejaculation, microsurgical epididymal sperm aspiration (MESA), percutaneous epididymal sperm aspiration (PESA), or testicular sperm extraction (TESE). In addition, indications for ICSI include idiopathic infertility and repeated conventional in vitro fertilization (IVF) failures [1]. Fertilization rate after ICSI is at about 70 to $80 \%$ in all ages combined [2]. This suggests that, despite injecting sperm into mature oocytes, failed fertilization still occurs. Total failed fertilization (TFF) refers to failure of fertilization in all mature oocytes and "failed fertilization" refers to failure of fertilization in any mature oocyte. Based on a considerable emotional and financial involvement in a cycle of assisted reproduction, TFF is a distressful event for the infertile couple as well as the fertility professionals. TFF occurs in $5-10 \%$ of IVF [3] and 1-3\% of ICSI cycles [4]. TFF after ICSI cycles is mostly due to low number of mature oocytes [4] or oocyte activation failure [5]. TFF is a rare event in cases with normal oocytes and sperm [6]. Some patients may face repeated TFF in spite of normal sperm parameters and good ovarian response [7]. In such cases, the primary reason for failed fertilization after ICSI is lack of oocyte activation, as more than $80 \%$ of these oocytes contain a sperm [4]. Considerable advances in artificial oocyte activation and recovery of sperm from epididymis or testis, suitable for ICSI, help avoid TFF. This chapter discusses the factors affecting success rate of ICSI, highlights causes of failure and suggests remedies for failed fertilization after clinical ICSI.

\section{Procedural effects of ICSI technique}

The risk of oocyte damage by the ICSI procedure is low in humans and is due to both the skill of the person performing the injection procedure and the quality of the gametes used 
during the procedure [8]. The embryologist performing ICSI procedure is a significant predictor of fertilization, and laboratory conditions (i.e. incubators, culture of oocytes individually versus grouped) do not affect the rates [9]. When fertilization failure in most or all of the injected oocytes occurs, with experienced practitioners using normal sperm, the diagnosis falls to oocyte dysfunction, oocyte activation failure, or inability of sperm to be decondensed and processed by the oocyte.

Although ICSI is now a routine, it remains a very demanding technique to master, due partly to its inherent technical difficulty and partly to the heterogeneity of the cases. It is generally agreed that the ICSI procedure is subject to a "learning curve" [9] and that one common technical failure is not depositing the sperm within the oocyte cytoplasm. In this situation, the oocyte membrane may not have been broken during attempts to aspirate the ooplasm into the ICSI needle. Thus, the sperm is deposited next to the membrane so that when the oolemma returns to its original position, the sperm is pushed out into the perivitelline space, or is trapped inside a sac formed by the membrane [10]. The sperm may also become adherent to the tip of the injection needle or remain within the injection needle and be inadvertently pulled out upon withdrawal of the needle from the cytoplasm. Aspiration of the ooplasm is always used to make sure that the oocyte membrane is broken during injection. However, if the ooplasm is aspirated too much, degeneration of the oocyte frequently results. The degeneration of oocytes after ICSI is often a result of a fault in the ICSI technique, e.g. an injection pipette that is too large, not positioned properly or not sharp enough. Figure 1 shows different stages of egg maturation and damaged oocytes after ICSI and Figure 2 shows normal and abnormal fertilization after ICSI.
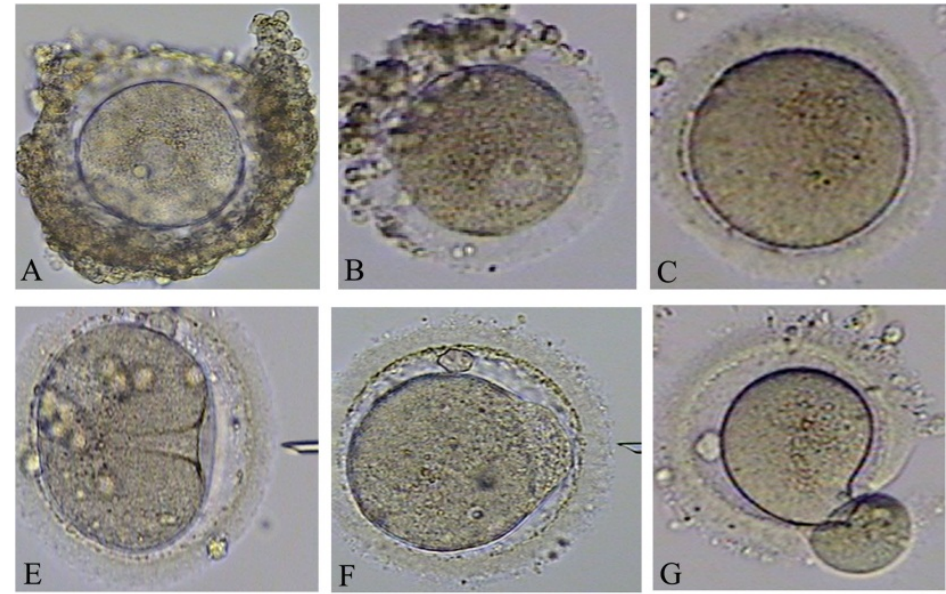
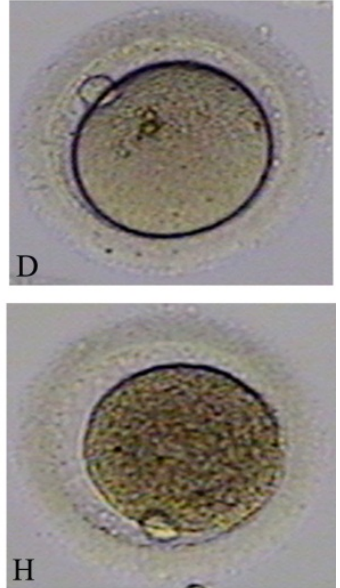

Figure 1. First row: $A$ and $B$ are GV, $C$ is $M I$ and $D$ is MII oocyte. Second row: $E$ shows typical funnel that appears after ICSI, F shows leakage of ooplasm after ICSI, G shows oocyte damage during denudation and $\mathrm{H}$ is an atretic oocyte after ICSI. 

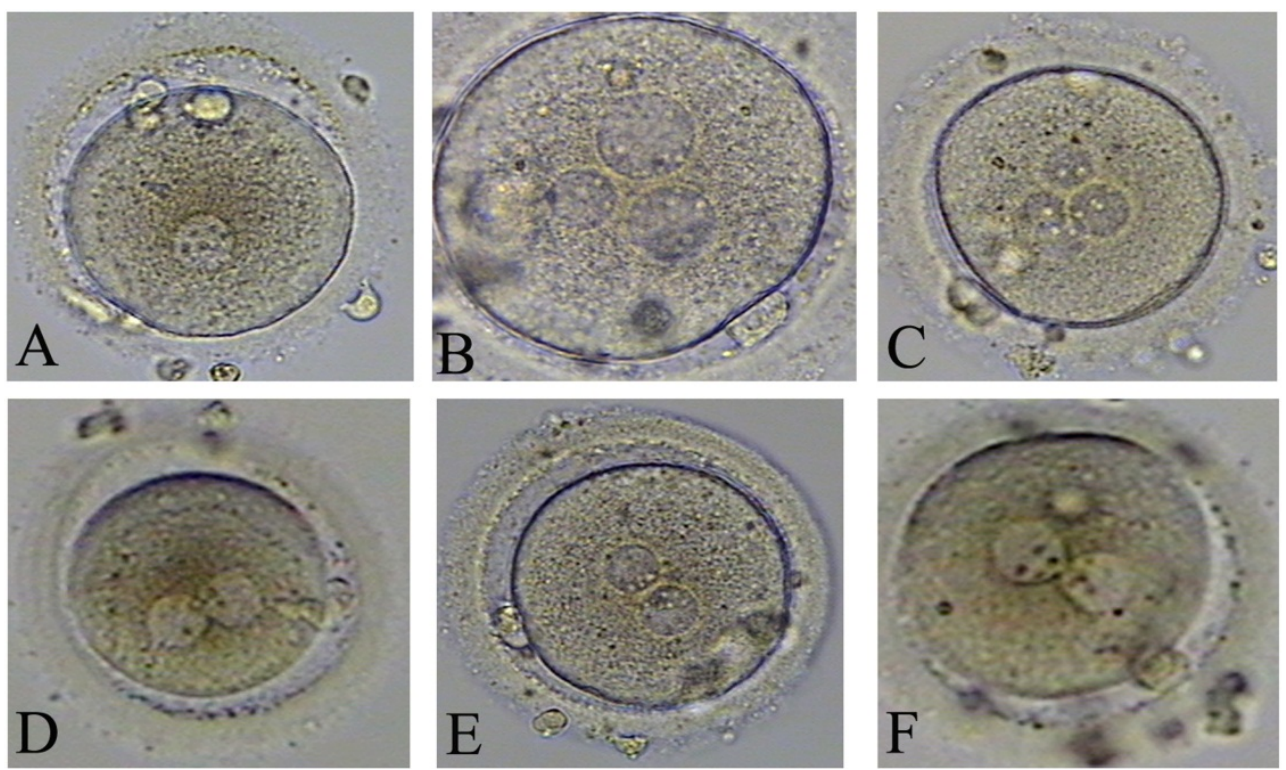

Figure 2. First row: $A$ is an egg with1 pronucleus (PN), B with 3 and $C$ with $4 \mathrm{PN}$. All these are abnormal fertilizations. Second row: Oocyte in D, E or F, each has 2 PN. This is a sign of normal fertilization.

Proper orientation of the polar body and needle position are also important, since improper positioning can damage or disrupt the metaphase plate during needle entry. In addition, disturbances in the nuclear spindle may dispose oocytes to aneuploidy or maturation arrest. Thus, perturbation of the cytoskeletal integrity of oocyte may critically influence the fate of the embryo. During ICSI, the location of the first polar body is commonly used as an indication of the spindle position, with the assumption that they are located in close proximity. To avoid damage to the spindle, oocytes are injected at the 3 o'clock position with the first polar body at the 6 or 12 o'clock position. However, with the aid of the computer assisted polarization microscopy, some reports suggest that the location of the first polar body does not necessarily correspond to the spindle position [11, 12]. The reasons for the displacement of the spindle are not fully understood [13]. Further detail on this aspect is given under use of Polscope.

Injection of a motile sperm without immobilization leads to poor fertilization rates [14]. In such cases, sperm with moving tails can be seen in the oocyte and sperm-oocyte interaction is obstructed by the normal sperm plasma membrane. Damage to the sperm membrane is necessary for successful oocyte activation following ICSI, as it induces gradual disruption of other parts of the sperm membrane allowing entry of sperm nucleus decondensing factor of the oocyte to induce initial swelling of the head. Because of this swelling, the sperm plasma membrane ruptures and sperm-associated oocyte activating factors are released into the ooplasm and induce oocyte activation. A modified ICSI technique is characterized by 
pushing the needle tip close to the membrane opposite the puncture site, aspirating the cytoplasm at this point and releasing the sperm in the centre of oocyte [5]. This modification improves fertilization in oocyte-dependent activation failure, but its routine application does not improve the overall results.

\section{Use of PICSI}

The cell surface hyaluronic acid (HA) binding glycoprotein is present in spermatozoa of different species including rat, mice, bull, and human [15]. The formation of hyaluronic-acid (HA)-binding sites on the sperm plasma membrane is one of the signs of sperm maturity. Various biochemical sperm markers indicate that human sperm bound to HA exhibit attributes similar to that of zona pellucida-bound sperm, including minimal DNA fragmentation, normal shape, and low frequency of chromosomal aneuploidies [16].

PICSI Sperm Selection Device (Biocoat, Inc. Horsham, PA, USA) offers advantage in selecting sperm for ICSI. The PICSI device, a dish similar to ICSI dish, contains 3 microdots of hyaluronan hydrogel which need to be hydrated by media before ICSI. The prepared sperm sample is placed at the edge of the microdrop of PICSI dish. Mature, biochemically competent sperm bind to the hyaluronan where they can be isolated by the embryologist and used for ICSI (Figure 3). The research supports that hyaluronan-bound PICSI-selected sperm are, in the vast majority of cases, more mature, exhibit less DNA damage, and have fewer chromosomal aneuploidies [17]. Further studies are needed to prove that use of PICSI technique improves pregnancy rates and reduce the number of IVF miscarriages.
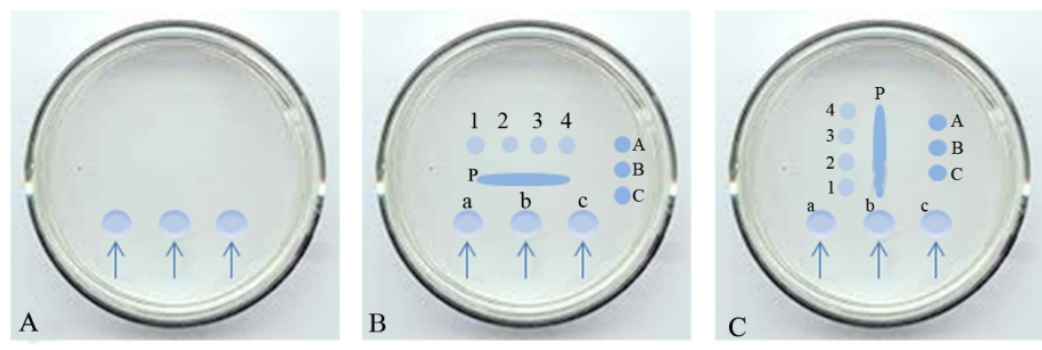

Figure 3. A is a graphical presentation of a PICSI dish. Each arrow is pointing to a dot containing hyaluronan. B and C are suggested arrangements for oocyte washing, PVP and ICSI drops (A, B and C are oocyte washing drops, $\mathrm{P}$ is PVP drop, $\mathrm{a}, \mathrm{b}$ and $\mathrm{c}$ are hyaluronan dots and1, 2, 3, and 4 are ICSI drops).

\section{Use of IMSI}

Intracytoplasmic morphologically selected sperm injection (IMSI) is examination of unstained spermatozoa at 6000 or higher magnification to select sperm with best morphology. It is based on a method of high magnification motile sperm organelle morphology examination (MSOME) [18]. It requires an inverted light microscope equipped 
with high power Nomarski optics enhanced with digital imaging. Such examination helps to identify spermatozoa with a normal nucleus and nuclear content [19]. The exact indications of IMSI [20] and usefulness [21] are debatable. Finding normal-looking spermatozoa took a minimum of $60 \mathrm{~min}$, and up to $210 \mathrm{~min}$, depending on the quality of the semen sample. The technique required two embryologists working together on the analysis of the same sample at the same time in order to minimize the subjective nature of sperm evaluation [22]. The IMSI procedure improved embryo development and the laboratory and clinical outcomes of sperm microinjection in the same infertile couples with male infertility and poor embryo development over the previous ICSI attempts [23]. A successful childbirth after IMSI without assisted oocyte activation in a patient with globozoospermia has been reported [24]. Randomized large-scale trials are needed to confirm the beneficial effects of IMSI in couples with poor reproductive prognoses [25].

\section{Use of polscope}

The oocyte spindle can be imaged non-invasively based on the birefringence, an inherent optical property of highly ordered molecules, such as microtubules, as they are illuminated with polarized light. Polarized light microscopy has been applied to embryology for decades. A digital, orientation-independent polarized light microscope, the Polscope, has demonstrated the exquisite sensitivity needed to image the low levels of birefringence exhibited by mammalian spindles [26]. The Polscope, is used to protect the meiotic spindle from damage during ICSI. The oocytes having Polscope visualised spindle have higher fertilization rate. When the spindle is located at $0^{\circ}-30^{\circ}$ in relation to the first polar body, ICSI achieves highest fertilization rate [27]. The use of Polscope is still not widely practiced and further improvements are needed. Morphometric evaluation of the spindle through the Polscope is not consistent with confocal analysis. This suggests that the Polscope may still be a rather inefficient method for assessing the metaphase II spindle [28].

\section{Timing of ICSI}

Both nuclear and cytoplasmic maturation of oocytes have to be completed to ensure optimal conditions for fertilization. Oocytes are retrieved prior to ovulation for IVF or ICSI procedures. In the pre-ovulatory phase, meiotic division of the oocyte must progress to metaphase II which is considered nuclear maturation and is evident by extrusion of first polar body. The oocyte also must develop the capacity to attain fertilization and initiate embryo development which is considered cytoplasmic maturation $[29,30]$. Cytoplasmic maturity is thought to be asynchronous with nuclear maturity in stimulated cycles [31, 32] and the fertilizing ability of an oocyte with a mature nucleus is not necessarily at its maximum potential. Thus, preincubation of oocytes prior to IVF or ICSI may induce cytoplasmic maturation that could eventually increase fertilization and also pregnancy rates. The human oocytes progressively develop the ability for full activation and normal development during the MII arrest stage [33]. 
The ICSI technique is generally similar among different centres but the time intervals from retrieval to denudation and from denudation to ICSI varies. Very few studies have addressed this aspect, with discrepancies in the conclusions [34, 35]. The preincubation period between oocyte retrieval and injection improves the percentage of mature oocytes $[36,37]$, the fertilization rate [35, 37], and the embryo quality [35]. The appropriate incubation time for mature oocytes before ICSI is 5-6 h. This time improves embryo quality and pregnancy rate in ICSI cycles. The maximum clinical pregnancy rate is observed when ICSI is performed $5 \mathrm{~h}$ after oocyte retrieval. The clinical pregnancy rate dropped significantly when ICSI was performed $6 \mathrm{hrs}$ after oocyte retrieval (Falcone et al., 2008). A longer oocyte pre-incubation (9- 11 hours) prior to ICSI is thought to have detrimental effects on embryo quality [38], probably due to oocyte ageing.

\section{Sperm related factors}

\subsection{Sperm structural defects}

Normal sperm ultrastructure correlates with positive IVF results [39]. Single structural defects involving the totality of ejaculated sperm are among rare cases of untreatable human male infertility. This form of infertility is of genetic origin and is generally transmitted as an autosomal recessive trait. Numerous defective genes are potentially involved in human isolated teratozoospermia but such defects have not been defined at the molecular level in most cases [40]. An in-depth evaluation of sperm morphology by transmission electron microscopy (TEM) can improve the diagnosis of male infertility and can give substantial information about the fertilizing competence of sperm [41, 42]. The TEM evaluation of sperm can also identify potentially inheritable genetic disorders (for example primary ciliary dyskinesia, Kartagener's syndrome), providing valuable information for couples contemplating ICSI [43].

Abnormal spermatozoa with head vacuoles account for the patient infertility. Sperm head vacuoles are easily detectable in human spermatozoa under the electron microscope. A sperm head vacuole is considered abnormal when it exceeds $20 \%$ of the head's crosssectional area. In rare cases, primary spermatozoa deformity is $100 \%$ vacuolated head [44]. There is a strong correlation between high relative vacuole area to sperm head and poor sperm morphology [45]. No correlation is observed between DNA defect and sperm-head morphology [46]. However, macrocephalic and large-headed spermatozoa are commonly associated with a low chance of pregnancy, mainly in relation to meiotic abnormalities during spermatogenesis. Enlarged-head spermatozoa are linked to sperm chromatin condensation dysfunction with no major meiotic dysfunction [47].

Acrosome agenesis is most often associated with a spherical shape of the head and is defined as "round head defect" or "globozoospermia". The underlying causes of the syndrome remain to be elucidated [48]. The genetic contribution has been postulated as well [49]. An additional case report [50] supports it. Studies show that the pathogenic genes associated with globozoospermia include SPATA16, PICK1, GOPC, Hrb, Csnk2a2 and bs 
[51]. Globozoospermia results from perturbed expression of nuclear proteins or from an altered golgi-nuclear recognition during spermiogenesis. The sperm show both gross and ultrastructural abnormalities, including the complete lack of an acrosome, abnormal nuclear membrane and mid-piece defects. Depending on the severity of the defect, the fertilization rate after ICSI with round headed sperm ranges from $0 \%$ to $37 \%$ [52, 53]. Successful pregnancies have been reported after ICSI in patients with globozoospermia with or without oocyte activation $[54,53,55]$. The most likely cause for failed fertilization after ICSI using round-head sperm is inability of sperm to activate the oocyte. In some forms of globozoospermia, arrest of nuclear decondensation and/or premature chromosome condensation also causes fertilization failure [55].

\subsection{Sperm DNA damage}

DNA damage in the male germ line is associated with poor fertilization rates following IVF, defective pre-implantation embryonic development and high rates of miscarriage and morbidity in the offspring, including childhood cancer [56, 57]. Activation of embryonic genome expression occurs at the four to eight-cell stage in human embryos [58], suggesting that the paternal genome may not be effective until that stage. Therefore, a lack of correlation between elevated DNA strand breaks in sperm and fertilization rates may occur before the four to eight-cell stage [59, 60]. Many published articles indicate that DNA strand breaks are clearly detectable in ejaculated sperm and their presence is heightened in the ejaculates of men with poor semen parameters [61, 62]. Nuclear DNA damage in mature sperm includes single strand nicks and double strand breaks that can arise because of errors in chromatin rearrangement during spermiogenesis, abortive apoptosis and oxidative stress $[63,64]$. In the same individuals, testicular samples show a significantly lower DNA damage compared to ejaculated spermatozoa $(14.9 \% \pm 5.0$ vs. $40.6 \% \pm 14.8, \mathrm{P}<0.05)$, but significantly higher aneuploidy rates for the five analyzed chromosomes $(12.41 \% \pm 3.7$ vs. $5.77 \% \pm 1.2$, $\mathrm{P}<0.05)$. While testicular spermatozoa appear favourable for ICSI in terms of lower DNA damage, this potential advantage could be offset by the higher aneuploidy rates in testicular spermatozoa [65].

Two tests are most commonly reported as indicators of sperm nuclear integrity; terminal deoxynucleotidyl transferase-mediated dUTP nick end labeling (TUNEL) and sperm chromatin structure assay (SCSA). The TUNEL technique labels single or double-stranded DNA breaks, but does not quantify DNA strand breaks in a given cell. The SCSA, a quantitative and flowcytometric test, measures the susceptibility of sperm nuclear DNA to acid-induced DNA denaturation in situ, followed by staining with acridine orange [66]. The SCSA accurately estimates the percentage of sperm chromatin damage expressed as DNA fragmentation index (DFI) with a cut-off point of 30\% to differentiate between fertile and infertile samples [67]. A statistically significant difference is seen between the outcomes of ICSI versus IVF when DFI is $>30 \%$ [68]. The biological explanation behind the superior results of ICSI in cases of high DFI needs to be elucidated. One possibility may be that women undergoing ICSI, on average, produce healthier oocytes with a better DNA repair capacity than women undergoing IVF, as in the ICSI group infertility is mainly caused by male factor. 
Other tests of sperm nuclear DNA integrity include in situ nick translation and the comet assay. The toluidine blue and sperm chromatin dispersion test are potential new assays [69]. At present, there are two major strategies that may be considered for the treatment of men exhibiting high levels of DNA damage in their sperm: (i) selective isolation of relatively undamaged sperm and (ii) antioxidant treatment [70]. The lack of consensus in defining a clinically relevant standard DNA fragmentation test with a meaningful cut-off level brings challenges in implementing the routine use of sperm DNA integrity assessment in daily practice [71].

\section{Injection with immature sperm}

Round spermatid nucleus injection (ROSNI) or round spermatid injection (ROSI) is a method in which precursors of mature sperm obtained from ejaculated specimens or testicular sperm extraction (TESE) are injected directly into oocytes. ROSNI is proposed as a treatment for men in whom other more mature sperm forms (elongating spermatids or sperm) cannot be identified for ICSI [72]. It is not widely performed, not as successful as ICSI and is still an experimental procedure. It should be applied only in the setting of a clinical trial approved and overseen by a properly constituted institutional review board. Accurate identification of round spermatid is a technical challenge of ROSNI. It is difficult to distinguish haploid round spermatids from diploid spermatogenic precursors and somatic cells using the standard optics present in most clinical IVF laboratories. Mouse round spermatids have increased levels of DNA fragmentation [73] that may interfere with fertilization [63]. Increased DNA damage may occur because of deficient sperm nuclear protamine to histone replacement and decreased nuclear condensation in these immature sperm allowing increased susceptibility to reactive oxygen species and other damaging agents in culture. Another major concern is genetic risk. Any genetic abnormality sufficiently severe to result in meiotic arrest during spermatogenesis may also have adverse effects on other normal cellular processes or other systemic manifestations. Occurrence of significant congenital anomalies in ROSNI-conceived pregnancies raises serious concerns [74]. ROSNI should not be performed when more mature sperm forms (elongating spermatids or sperm) can be identified and used for ICSI. Patients who may be candidates for ROSNI should receive careful and thorough pre-treatment counselling to ensure they are clearly informed of the limitations and potential risks of the procedure [75].

\section{Premature chromosomal condensation}

When a cell, with chromosomes in MII, fuses with an inter-phase cell, the nuclear membrane of the cell in the inter-phase dissolves and its chromatin condenses. This phenomenon is called premature chromosomal condensation (PCC) [76]. Following penetration of sperm into an oocyte; oocyte activation is triggered, resulting in completion of meiosis and formation of both male and female pronuclei. Under some circumstances although the sperm is within the oocyte, fertilization fails to occur, the oocyte remains in the MII stage and the sperm head transforms into PCC, separate from the oocyte chromosomes [77, 78]. Chromatin analysis of human oocytes has revealed that sperm PCC is one of the prevalent causes of fertilization failure in both IVF and ICSI [77]. 
It is not yet fully understood how the sperm activates the oocytes. The failure of fertilization after ICSI may result from either the lack or deficiency of activating factors in sperm or from the lack of ooplasmic factors triggering sperm chromatin decondensation [79, 80]. Several pieces of evidence point to PLC $\zeta$ being the physiological agent of oocyte activation and is detectable in different localities within the sperm head: the equatorial segment and acrosomal/post-acrosomal region [81].

During normal spermiogenesis, $85 \%$ of histones are replaced with protamines [82], which results in sperm chromatin condensation. A sperm with a condensed nucleus is in the G1 stage when entering an MII oocyte and is protected from PCC because an active maturationpromoting factor (MPF) is not capable of reacting with protamine-associated DNA. Once sperm nuclear decondensation factors from the ooplasm enter the sperm, the sperm head swells and sperm associated oocyte activating factor is released. This results in MPF inactivation [83], the completion of meiosis 2 and the oocyte enters the G1 stage. During this time, protamines are slowly replaced by histones and cell cycle synchronization takes place. Under some circumstances, the oocyte fails to activate and remains arrested at MII. Because of the presence of an active MPF, sperm chromatin transforms into condensed chromatin. Sperm with excessive histones are prone to PCC.

Sperm PCC has been associated with the type of ovarian stimulation protocol. Some protocols, such as clomiphene citrate and human menopausal gonadotropin stimulation may tend to recruit immature oocytes with immature cytoplasm [84]. Immature cytoplasm is believed to make sperm susceptible to a high incidence of PCC after insemination because of the inability of these immature oocytes to undergo oocyte activation [85]. The incidence of sperm PCC reported in the literature ranges from 10.1 to $85 \%$ [86, 87], with higher values noted in cases of round headed sperm injection as they fail to activate the oocyte. Furthermore, other studies suggest a correlation between fertilization outcome post-ICSI and percentage of sperm with protamine deficiency [88]. The effect of sperm protamine deficiency on fertilization rate emphasizes the need for accurate sperm selection during ICSI as protamine-deficient sperm, in the form of slightly amorphous head, may find the chance of being injected due to inappropriate sperm selection [88].

\section{Sperm motility and progression}

Defective sperm tail is the principal cause of sperm motility disorders. There are two main forms of tail disorders with different phenotypic characteristics and consequences for male fertility: non-specific tail anomalies and various genetic disorders including primary ciliary diskinesia and the dysplasia of the fibrous sheath [89]. In non-specific tail anomalies, ICSI has good prognosis and does not pose additional risks in view of the lack of recognized genetic components in this Disorder. Significant sperm abnormalities of proven or suspected genetic origin are rare conditions responsible for extreme asthenozoospermia or total sperm immotility. Affected patients complain of male infertility and chronic respiratory disease, alterations caused by abnormal function of sperm flagella and respiratory cilia. In patients with tail genetic disorders, ICSI results in normal rates of fertilization and implantation, and 
many births of healthy babies have been reported. The main concern that remains is the potential transmission to the offspring [89].

Whether sperm movement is slow or rapid generally has no influence on ICSI results. However, injection of immotile sperm usually results in impaired fertilization. In particular, where a non-viable immotile sperm is injected into an oocyte, normal fertilization and pregnancy rarely occurs $[90,91]$. In case of immotile sperm, it is possible that the sperm may be dead. The most common practice to select viable non-motile sperm for ICSI involves the hypo-osmotic swelling (HOS) test. However, preliminary results in animal experiments (mouse and rabbit) indicate that viability of injected sperm is not an absolute pre-requisite for fertilization. Embryos derived after injecting mouse oocytes with freeze-dried and thawed sperm developed normally [92]. It appears that provided the DNA integrity of the sperm is maintained, embryos can be generated, at least in animal model, from severely damaged sperm that are no longer capable of normal physiological activity.

The identification of a viable spermatozoon amongst immotile spermatozoa for ICSI often is difficult. However, selection of birefringent spermatozoa under Polscope shows promising results in asthenozoospermic men and men undergoing testicular sperm aspiration or extraction before ICSI [93]. The other tests employed are hypo-osmotic swelling test, the stimulation of motility with pentoxyfilline and non-contact diode laser [94, 95, 96, 97, 98].

In patients with $100 \%$ immotile sperm, HOS test is a useful method to examine sperm viability. It measures the functional integrity of the sperm membrane [99]. Upon exposure of the sperm to hypo-osmotic conditions, the intact semi-permeable barrier formed by the sperm membrane allows an influx of water and results in swelling of the cytoplasmic space and curling of the sperm tail fibers. Only viable sperm react to the HOS solution since dead sperm are unable to maintain the osmotic gradient.

Sperm HOS test based on fructose and sodium citrate dihydrate is applied for identification of immotile sperm for ICSI [100]. A significantly greater fertilization and cleavage rate after injection of sperm selected using the HOS test is achieved in contrast to injection of randomly selected sperm. A modified $\mathrm{HOS}$ test based on $\mathrm{NaCl}$ solution further improves fertilization rate in patients with 100\% immotile sperm [101]. In these procedures, approximately 200,000 sperm are exposed to the HOS solution for 1 hour at $37^{\circ} \mathrm{C}$. A modified HOS test has been used for samples with a low sperm count such as testicular samples [102]. In this technique, individual morphologically normal sperm is aspirated by microinjection pipette and is exposed to HOS solution for a brief period to minimize the sperm membrane damage.

A mixture of $50 \%$ culture medium and $50 \%$ deionized grade water has the least delayed harmful effects on sperm vitality [103]. This mixture achieves similar implantation, pregnancy and ongoing pregnancy rates in the ejaculated and testicular non-motile sperm groups [104]. It is a simple and practical procedure and achieves acceptable and comparable pregnancy rates. 
Obtaining viable spermatozoa from testicular biopsies using pentoxifylline is more effective in terms of fertilization and pregnancies than obtaining it through an HOS test [97]. The clinical use of pentoxifylline for activation of immotile ejaculatory sperm before ICSI in patients with Kartagener's syndrome improves the outcome of the treatment and reduces the need of invasive intervention such as TESE in these patients. The immotile sperm are treated for $30 \mathrm{~min}$ with pentoxifylline $(1.76 \mathrm{mM})$ before ICSI. Some spermatozoa show minimal motion and can be used for ICSI. Fertilization rate after ICSI is about 75\% [105].

\section{Sperm origin}

A new era in the field of assisted reproduction opened after the achievement of pregnancies and births after ICSI of human oocytes [106]. In special cases of long-standing male infertility, only a few functional sperm are available. By means of ICSI, most sub-fertile men and even men previously considered sterile (those with azoospermia, extreme oligozoospermia or cryptozoospermia) can now father a child.

Azoospermia, is the most severe form of male factor infertility. The condition is currently classified as 'obstructive' or 'non-obstructive'. Obstructive azoospermia is the result of obstruction in either the upper or lower male reproductive tract. Sperm production may be normal but the obstruction prevents the sperm from being ejaculated. Non-obstructive azoospermia is the result of testicular failure where sperm production is either severely impaired or nonexistent, although in many cases sperm may be found and surgically extracted directly from the testicles [107].

Conflicting results for fertilization and pregnancy rates are available in the literature after use of ejaculated or surgically retrieved sperm. After ICSI, ejaculated or surgically extracted sperm, when motile and morphologically normal, result in similar fertilization, implantation $[108,109]$ and clinical pregnancy rates ([109]. The incidence of early or late spontaneous abortion and ectopic pregnancy, or malformations is also similar [108]. However, after conventional IVF, even testicular or epididymal aspirates with very good sperm concentration and motility, generally achieve low fertilization and pregnancy rates [110].

The effect of cryopreservation of sperm on ICSI outcome has been thoroughly studied. Current studies suggest that the use of fresh or frozen-thawed sperm does not appear to affect ICSI outcomes [111]. Testicular tissue and epididymal sperm can be cryopreserved successfully without markedly reducing subsequent fertilization and implantation rates and repeated testicular biopsy can be avoided without the risk of any decrease in the outcome [112].

The origin of the sperm used in ICSI does not have a major influence on the early life outcomes for the offspring, but transgenerational and epigenetic effects remain unknown. From the limited information available, it appears that there is no increased risk of congenital malformations in children born from ICSI. There is, however, a small increase in both de novo and inherited chromosome abnormalities. In terms of growth and neurodevelopment, there are very few studies, and so far, no adverse outcomes have been found in young children whose fathers have a sperm defect [113]. 


\section{Oocyte related factors}

\subsection{Oocyte activation}

Oocyte activation is a complex series of events that results in the release of the cortical granules, activation of membrane bound ATPase, resumption of meiosis with the extrusion of the second polar body and finally the formation of male and female pronuclei. The ovulated or retrieved oocyte activates when the sperm enters, by either natural penetration or ICSI. In cases of oocyte activation failure, artificial means of oocyte activation are helpful.

The oocytes remain arrested at MII if maturation has been completed. When one sperm contacts the oolemma and penetrates into the ooplasm, intracellular calcium oscillation occurs [114]. This increase in the concentration of calcium underlies oocyte activation and initiation of development. In mammals, growing experimental evidence supports the notion that, following fusion of the gametes, a factor from sperm is responsible for inducing calcium oscillations and stimulating inositol 1,4,5-trisphosphate (IP3) production [115]. Initial evidence stemmed from injection of cytosolic sperm extracts into oocytes that reproduced the calcium responses associated with fertilization regardless of the species of origin [116, 117]. Subsequent biochemical characterization of the extracts revealed that the active component contained a protein moiety [116] that possessed phospholipase C (PLC) like activity capable of inducing production of IP3 $[118,119]$ and that the PLC activity was highly sensitive to calcium [120]. A screen of expressed sequenced tags from testes identified a sperm-specific phospholipase C, PLC $\zeta$. The presence of PLC $\zeta$ correlates with calcium activity in cytosolic sperm extracts [121]. Moreover, injection of oocytes with the recombinant protein [122] or with the encoding mRNA induces fertilization-like oscillations (Saunders, 2002), whereas depletion of PLC $\zeta$ from the extracts with specific antisera abrogates PLC $\zeta$ activity [123] and the calcium oscillatory activity of the extracts [121, 123]. The PLC $\zeta$ is located in the equatorial region of human sperm. Men whose sperm are unable to initiate calcium oscillations consistently fail to fertilize following ICSI and lack PLC $\zeta$ [124]. It has to be established that PLC $\zeta$ is the sole calcium oscillation-inducing factor and how its absence has an impact on male fertility.

The process of natural fertilization encompasses the entry of the sperm, oocyte activation and the first mitotic division resulting in a 2 cell embryo. Two steps are important for successful fertilization following ICSI, namely immobilization of the sperm and rupture of the oolemma in order to facilitate the liberation of the cytosolic sperm factor responsible for the oscillator function [14].

Low fertilization rates after ICSI in patients with round-headed sperm, globozoospermia, is a result of reduced ability of round-headed sperm to activate the oocyte. In the literature, the success rates of ICSI in cases of globozoospermia are variable. Assisted oocyte activation combined with ICSI may overcome the infertility associated with globozoospermia. Normal healthy live birth without assisted oocyte activation has also been achieved [54]. Apart from low fertilization rates associated with the use of round-headed sperm, cleavage rates are also compromised and these sperm may lack normal centrosomes [52]. Assisted oocyte 
activation and ICSI restore fertilization, embryo cleavage and development for patients with globozoospermia [125].

Assisted oocyte activation after ICS is very efficient in patients with a suspected oocyterelated activation deficiency and previous total fertilization failure after conventional ICSI. However, when there was a prior history of low fertilization, one should be careful and test the efficiency of assisted oocyte activation on half of the sibling oocytes, because assisted oocyte activation is not always beneficial for patients with previous low fertilization and a suspected oocyte-related activation deficiency. For these patients, a split assisted oocyte activation-ICSI cycle using sibling oocytes can help to distinguish between a molecular oocyte-related activation deficiency and a previous technical or other biological failure [126].

Assisted oocyte activation aims to mimic the action of sperm penetration [127]. Some assisted activation treatments such as strontium chloride [128] and ionomycin [129], promote an increase in intracellular free calcium concentrations by the release of calcium from cytoplasmic stores. Others such as electrical stimulus promote influx of calcium from the extracellular medium and some treatments such as ethanol promote both effects [129].

A birth after oocyte activation by treatment with the calcium ionophore A23187 and ICSI has been obtained in 1994 [130]. Human oocytes injected with round-headed sperm are activated following combination of calcium chloride injection and ionophore treatment. This activation is followed by an apparently normal completion of meiosis, male and female pronuclei formation, embryonic development and successful delivery of a healthy infant [53]. A combination of calcium ionophore A23187 with puromycin stimulates the unfertilized oocytes $20-68 \mathrm{~h}$ after ICSI. It results in an activation rate of $91.2 \%(31 / 34)$, a cleavage rate of $64.7 \%(22 / 34)$ and high-quality embryo rate of $44.1 \%$ (15/34). Nearly all activated embryos derived from $2 \mathrm{PN} / 2 \mathrm{~PB}$ had a normal set of sex chromosomes and developed normally [131]. Although calcium ionophore A23187 and puromycin do not appear to be cytotoxic to oocytes and result in pregnancies and the birth of healthy babies when low concentrations are used, the possible teratogenic and mutagenic activity of calcium ionophore A23187 and puromycin needs further investigation in animal models and in humans.

Treatment with $10 \mathrm{mM}$ strontium chloride for $60 \mathrm{~min}$, approximately $30 \mathrm{~min}$ after ICSI results in activation and fertilization of all injected oocytes [132], development of the embryos to the blastocyst stage and delivery in patients with repeated fertilization failure [133]. Physical and mental development of the children from birth to 12 months is normal [132]. However, further studies are required to substantiate the finding that strontium chloride treatment is an effective method of artificial oocyte activation.

An electrical field can generate micropores in the cell membrane of gametes and somatic cells to induce sufficient calcium influx through the pores to activate cytoplasm through calcium dependent mechanisms [134]. Mouse oocytes injected with secondary spermatocytes or spermatids are fertilized when stimulated by electroporation and developed into normal offspring when the resultant embryos are transferred to a recipient 
[135]. Clinical pregnancy and delivery after oocyte activation by electrostimulation in combination with ICSI in previously failed to fertilize oocytes has been obtained [80]. Electrical stimulation rescues human oocytes that failed to fertilize after ICSI and stimulates them to complete the second meiotic division, to form pronuclei and to undergo early embryonic development [136]. Although the fertilization rate issimilar regardless of the number of electrical pulses applied, subsequent embryo development is dramatically improved in those oocytes that received three electrical pulses [136]. The embryo formation rate in the electrically activated group is $80 \%$ compared to $16 \%$ in the control group [137]. Although the fertilization rate is significantly higher in the electroactivated group (68\%) as compared with that of the control $(60 \%)$, a higher miscarriage rate is reported in the electroactivated group (5 of 15 pregnancies) compared to the control (3 of 33) [6]. Like any other new assisted reproductive procedure, the impact of electrical activation on oocyte and embryo health must be evaluated in larger studies before this procedure can be considered for routine clinical purposes. Ideally, karyotyping or fluorescent in situ hybridization analysis should be performed to assess the incidences of aneuploidy and mosaicism in the resultant embryos.

\section{Poor ovarian response}

The definition of 'poor response' in the literature is often based on a combination of factors, including the number of mature follicles, the number of oocytes retrieved and the peak estradiol level [138]. The cut-off levels for the number of follicles or oocytes that define poor response vary widely from study to study. Some authors feel that the definition of poor response should also include the degree of ovarian stimulation used and that a low oocyte number is detrimental only when high total dose of follicle stimulating hormone (FSH) has been administered [139]. Various endocrine and ultrasonographic markers and dynamic tests to assess ovarian reserve have been evaluated. Such tests include basal FSH on cycle day 3, clomiphene citrate challenge test, inhibin B, oestrogen, anti-Mullerian hormone, antral follicle counts and ovarian volume. The success of each test can be measured against ovarian response or live birth rate per cycle [140]. However, none of these tests has demonstrated a reliable predictive value and for many women poor ovarian response is not discovered until the first IVF cycle.

Poor response to gonadotropin stimulation occurs more often in older women, but may also occur in young women, regardless of the endocrinologic profile [141]. Poor responders have a significantly lower pregnancy rate per retrieval compared to normal to high responders in the same age group [138]. Although it is possible to have normal embryos and pregnancy in younger poor responders, the fertilization rate and quality of embryos in older poor responders are always low and the chance of achieving pregnancy in these patients is low. Poor responders also have an increased cycle cancellation rate due to retrieval of few or no oocytes and/or TFF. One of the major contributing factors for TFF after ICSI is $\leq 3$ MII oocytes retrieved (Esfandiari et al., 2005a). The rate of fertilization failure increases as the number of injected oocytes decreases [142]. There is a higher chance of having no embryos for transfer and significantly lower pregnancy rates when less than five oocytes are 
retrieved compared to cases with $\geq 5$ oocytes [143]. Limited information is available on IVM of immature oocytes retrieved from poor responders in conventional stimulation IVF/ICSI cycles and IVM is not a viable alternative to cancellation of IVF cycles in such patients [10].

Fecundity significantly decreases with increasing maternal age [144]. In a classic study of the Hutterite women, sterility increased from just over $10 \%$ at 34 years old to over $85 \%$ by the age of 44 years [145]. In women, all germ cells are formed during fetal life. The population of germ cells appears to rise steadily from 600000 at 2 months post conception, reaching a peak of 6800000 at 5 months. By the time of birth, the number declines to 2000000 of which $50 \%$ are atretic. Of the 1000000 normal oocytes in the newborn infant, only 300000 survive to the age of 7 years [146]. Continuous loss of oocytes occurs through the physiological process of follicular growth and atresia throughout life [147].

The incidence of TFF increases with age [10]. Older women are more likely to undergo multiple cycles, have decreased number of oocytes retrieved and a lower number of embryos transferred [9].

\section{Oocyte maturity}

One of the major causes of TFF after ICSI is a low number of retrieved MII oocytes [10]. About $20 \%$ of retrieved oocytes from controlled ovarian stimulation cycles are immature, either at metaphase-I (MI) or germinal-vesicle (GV) stage in human IVF [35]. Some of these oocytes may extrude the first polar body during in vitro culture and can be injected in ICSI cycles. This may be a useful strategy for patients with low number of retrieved oocytes. However, embryos derived from immature oocytes do not efficiently translate into pregnancies and live births. Therefore, the clinical significance of using immature oocytes in stimulated cycles needs further investigation [148].

The injection of MI oocytes immediately after denudation results in a high degeneration rate due to increased fragility of the oolemma. The fertilization rate of retrieved MI oocytes that remained MI at the time of ICSI is lower than the fertilization rate of sibling retrieved MI progressing to MII in vitro ( $25 \%$ compared to $62.2 \%$, respectively). It is less than half when compared to the fertilization rate of retrieved sibling MII oocytes (69.5\%). A high rate of multinucleated oocytes is also found in fertilized MI oocytes injected immediately after denudation [148].

In cases of poor responders and in patients with an unsynchronized cohort of follicles, where the presence of immature oocytes is frequent after stimulation [149], the use of immature oocytes is important in order to increase the number of embryos obtained in each cycle. Based on the assumption that oocyte maturity is a pre-requisite for obtaining normal fertilization, attempts have been made to mature GV and MI oocytes in vitro [147]. Despite the use of varying culture techniques and different stimulation protocols, such IVM oocytes consistently have lower fertilization rates, frequent cleavage blocks and overall retarded cleavage rate compared with sibling MII oocytes [147, 150]. The limited number of transfer cycles makes it difficult to draw solid conclusions about the value of transferring these 
embryos. It should be noted that the immature oocytes collected in stimulated cycles have already been under stimulation with high doses of gonadotropins and are exposed to hCG before retrieval. The nuclear maturation, cytoplasmic maturation and ensuing developmental capacity of these oocytes may very well be different in comparison with immature oocytes collected from small antral follicles of unstimulated ovaries in the typical IVM procedure [151].

\section{Oocyte morphology}

Poor oocyte morphology is a major determinant of failed or impaired fertilization. Normal features of a healthy mature oocyte at Metaphase-II (MII) include presence of a polar body, a round even shape, light colour cytoplasm with homogenous granularity, a small perivitelline space without debris and a colourless zona pellucida. In denuded oocytes, it is possible to assess the morphology and the nuclear maturity but not the cytoplasmic maturity. The MII oocytes with apparently normal cytoplasmic organization may exhibit extra-cytoplasmic characteristics, such as increased perivitelline space, perivitelline debris and/or fragmentation of the first polar body, which may reduce developmental competence of the oocyte [152]. It is common that extra-cytoplasmic and cytoplasmic dysmorphism occur together in the same oocyte (Figure 4 and 5). The dysmorphic phenotypes, which arise early in meiotic maturation, may be associated with failed fertilization and aneuploidy, while those occurring later in maturation may cause a higher incidence of developmental failure [153, 154].
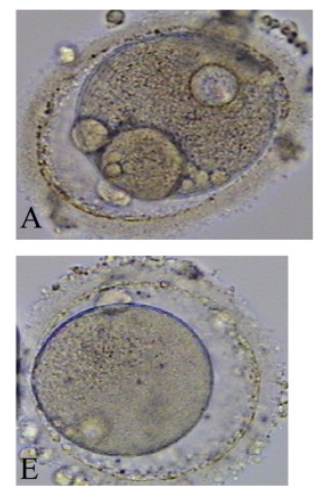
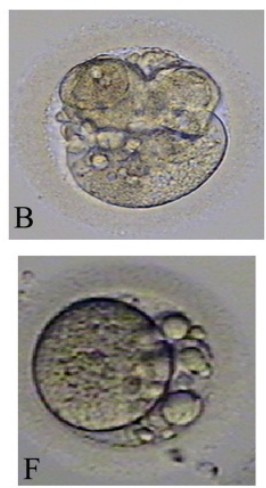
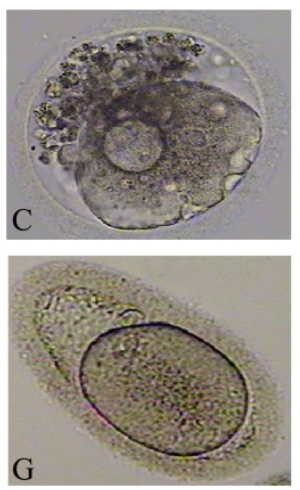
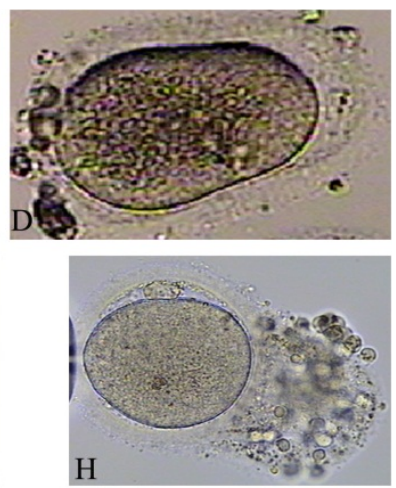

Figure 4. Oocytes in both rows show extra-cytoplasmic and cytoplasmic dysmorphism.

Decreased fertilization rates due to some oocyte dysmorphisms have been reported [152], while others failed to observe that association [155, 156, 157, 158, 159]. Lower pregnancy and implantation rates result when the transferred embryos originate from cycles with $>50 \%$ dysmorphic oocytes and the same dysmorphism repeats from cycle to cycle [155]. The repetitive organelle clustering is associated with an underlying adverse factor affecting the entire follicular cohort. The presence of a dark cytoplasm decreases by $83 \%$ the likelihood of obtaining good quality embryos [160]. However, another study did not find any adverse impact of dark colour of the oocytes on fertilization, embryo development and pregnancy 
rate [161]. In human oocytes, the cytoplasmic granularity can be homogeneous affecting the whole cytoplasm, or concentrated in the centre with a clear peripheral ring giving a darkened appearance to the cytoplasm [162]. The abnormal changes in the cytoplasm of MII oocytes may be a reflection of delayed cytoplasmic maturation that is unsynchronized with nuclear maturity [163].
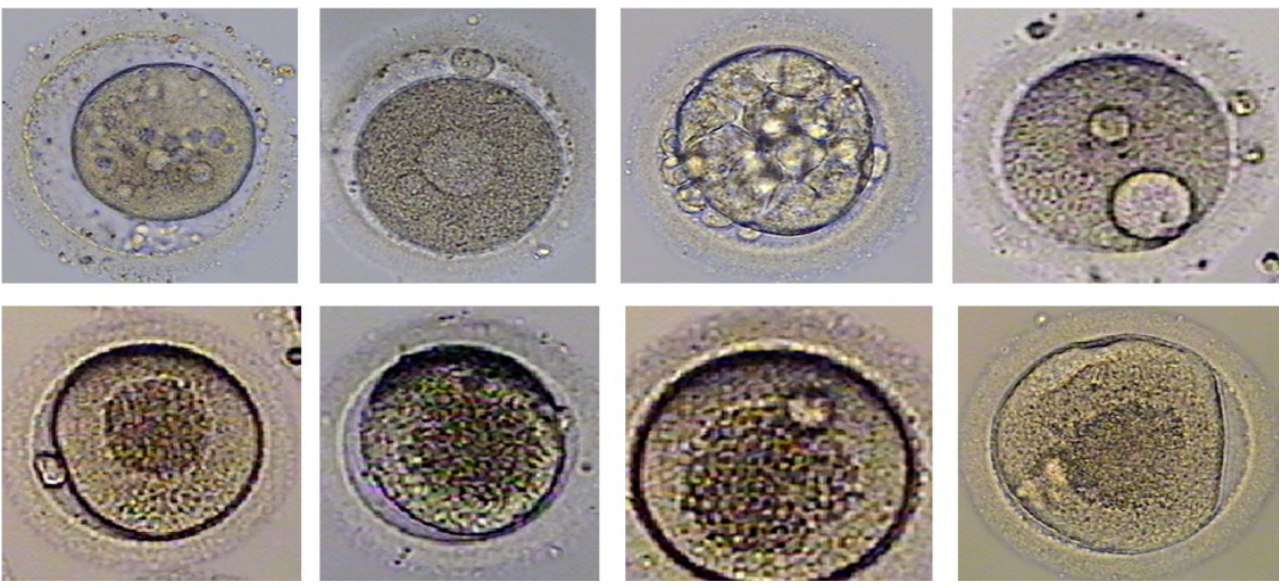

Figure 5. Oocytes in first row represent different degrees of vacuoles in cytoplasm. Each oocytes in second row has increased central granularity.

Normal fertilization, embryo development and live birth are possible after ICSI in oocytes with thick zonae, abnormal morphology or repeated polyspermia following conventional IVF. The oocytes with extreme morphological abnormalities should not be discarded as ICSI can result in fertilization, cleavage and normal embryonic development [164, 161]. The zonafree oocytes may be fertilized normally after ICSI and develop to the blastocyst stage [165]. Pregnancy in human [166] and live birth in mouse [167] and pig [168] have been obtained after transfer of embryos resulting from zona-free oocytes.

\section{ICSI after previous ICSI cycle failure}

Repeated ICSI treatment can be useful or necessary because there is a high possibility of achieving normal fertilization if a reasonable number of oocytes with normal morphology are available and motile sperm can be found. If there are no motile sperm present in the first ejaculate, a second sample should be required followed by PESA or TESE to obtain motile sperm. In this way, a sufficient number of motile sperm for ICSI are usually found in most men with severe asthenozoospermia.

A history of failed fertilization may be related to some gamete abnormality that may be modified or corrected at the next cycle. It has been documented that for a particular patient, fertilization results can be quite varied when followed through several ICSI cycles at the same centre [169]. The differences between fertilization rates are unexplained, although 
fluctuations in the gamete quality are probably contributory. Pretreatment endocrine assays and semen analyses prove to be of little value in forecasting failed fertilization. One-third of the patients with TFF achieved pregnancy with their own oocytes in a subsequent ICSI cycle [10]. Since follow-up ICSI treatment has been shown to result in fertilization in $85 \%$ of cases, repeated ICSI attempts are suggested in TFF $[4,170]$.

\section{Options for patients after repeated ICSI cycle failure}

Physicians should counsel patients based on the best possible evidence available and allow the couple to make an informed choice. The adverse result of a failed ICSI cycle does not imply a hopeless prognosis for future ICSI treatment. Very subtle improvements in semen parameters and/or oocyte yield/quality may result in fertilization in a subsequent ICSI attempt [169]. Otherwise, the options of donor sperm insemination, donated oocytes or embryos, adoption and remaining childless should be discussed with the couple [171].

\section{Conclusion}

Significant advances have been made in achieving fertilization, pregnancy and live birth in cases with severe male factor infertility, oocyte activation failure and ICSI technique. Usually fertilization is $80-100$ percent in mature eggs, however, low or no fertilization can still occur. Most cases of no fertilization occur due to very low number of mature oocytes, failure of oocyte activation or non-availability of appropriate sperm. Repeated ICSI attempts results in fertilization in $85 \%$ of cases.

\section{Author details}

Murid Javed* and Essam Michael

Astra Fertility Centre, Mississauga, Ontario, Canada

\section{References}

[1] Benavida CA, Julsen H, Siano L et al. 1999. Intracytoplasmic sperm injection overcomes previous fertilization failure with convention in vitro fertilization. Fertil Steril 72, 10411044.

[2] Palermo GD, Neri QV, Takeuchi T et al. 2009. ICSI: where we have been and where we are going. Sem Reprod Med 27,191-201.

[3] Mahutte NG, Arici A 2003. Failed fertilization: is it predictable? Curr Opin Obstet Gynecol 15, 211-218.

[4] Flaherty SP, Payne D, Matthews CD 1998. Fertilization failures and abnormal fertilization after intracytoplasmic sperm injection. Hum Reprod 13, 155-164.

[5] Ebner T, Moser M, Sommergruber M et al. 2004. Complete oocyte activation failure after ICSI can be overcome by a modified injection technique. Hum Reprod 19, 1837-1841.

${ }^{*}$ Corresponding Author 
[6] Mansour R, Fahmy I, Tawab NA et al. 2009. Electrical activation of oocytes after intracytoplasmic sperm injection: a controlled randomized study. Fertil Steril 91, 133-139.

[7] Tesarik J, Rienzi L, Ubaldi F et al. 2002. Use of a modified intracytoplasmic sperm injection technique to overcome sperm-borne and oocyte-borne oocyte activation failures. Fertil Steril 78, 619-24.

[8] Palermo GD, Cohen J, Alikani M et al. 1995. Intracytoplasmic sperm injection: a novel treatment for all forms of male factor infertility. Fertil Steril 63, 1231-1240.

[9] Shen S, Khabani A, Klein N et al. 2003. Statistical analysis of factors affecting fertilization rates and clinical outcome associated with intracytoplasmic sperm injection. Fertil Steril 79, 355-360.

[10] Esfandiari N, Javed MH, Gotlieb L et al. 2005. Complete failed fertilization after intracytoplasmic sperm injection - Analysis of 10 years data. Int J Fertil Women's Med 50, 187-192.

[11] Wang WH, Meng L, Hackett RJ et al. 2001. The spindle observation and its relationship with fertilization after ICSI in living human oocytes. Fertil Steril 75, 348-353.

[12] Wang WH, Meng L, Hackett RJ et al. 2001. Development ability of human oocytes with or without birefringent spindles imaged by Polscope before insemination. Hum Reprod $16,1464-1468$.

[13] Woodward BJ, Montgomery SJ, Hartshorne GM et al. 2008 Spindle position assessment prior to ICSI does not benefit fertilization or early embryo quality. Reprod BioMed Online16, 232-238.

[14] Vanderzwalmen P, Bertin G, Lejeune B et al. 1996 Two essential steps for a successful intracytoplasmic sperm injection: injection of immobilized spermatozoa after rupture of the oolema. Hum Reprod 11, 540-547.

[15] Ranganathan S, Ganguly AK, Datta K. 1994. Evidence for presence of hyaluronan binding protein on spermatozoa and its possible involvement in sperm function. Mol Reprod Dev. 38(1):69-76.

[16] Yagci A, Murk W, Stronk J, Huszar G. 2010. Spermatozoa bound to solid state hyaluronic acid show chromatin structure with high DNA chain integrity: an acridine orange fluorescence study. J Androl. 31(6):566-72. Epub 2010 Feb 4.

[17] Huszar G, Jakab A, Sakkas D, Ozenci CC, Cayli S, Delpiano E, Ozkavukcu S. 2007. Fertility testing and ICSI sperm selection by hyaluronic acid binding: clinical and genetic aspects. Reprod Biomed Online. 2007 May;14(5):650-63. Review.

[18] Bartoov B, Berkovitz A, Eltes F, Kogosowski A, Menezo Y, Barak Y. 2002. Real-time fine morphology of motile human sperm cells is associated with IVF-ICSI outcome. J Androl23, 1-8.

[19] Balaban B, Yakin K, Alatas C, Oktem O, Isiklar A, Urman B. 2011. Clinical outcome of intracytoplasmic injection of spermatozoa morphologically selected under high magnification: a prospective randomized study. Reprod Biomed Online. Feb 15. [Epub ahead of print]

[20] Vanderzwalmen P, Fallet C. 2010. IMSI: indications, results and reflexions. J Gynecol Obstet Biol Reprod (Paris). 39(1 Suppl):22-5. French. 
[21] Mauri AL, Petersen CG, Oliveire JB, Massaro FC, Baruffi LR, Franco JG Jr. 2010. Comparison of day 2 embryo quality after conventional ICSI versus intracytoplasmic morphologically selected sperm injection (IMSI) using sibling oocytes. Eur J Obstet Gynecol Reprod Biol 150:42-46.

[22] Antinori M, Licata E, Dani G, Cerusico F, Versaci C, D'angelo D, Antinori S. 2008. Intracytoplasmic morphologically selected sperm injection: a prospective randomized trial. Reprod Biomed Online 16:835-841.

[23] Knez K, Zorn B, Tomazevic T, Vrtacnik-Bokal E, Virant-Klun I. 2011. The IMSI procedure improves poor embryo development in the same infertile couples with poor semen quality: a comparative prospective randomized study. Reprod Biol Endocrinol. 29;9:123.

[24] Sermondade N, Hafhouf E, Dupont C, Bechoua S, Palacios C, Eustache F, Poncelet C, Benzacken B, Lévy R, Sifer C. 2011. Successful childbirth after intracytoplasmic morphologically selected sperm injection without assisted oocyte activation in a patient with globozoospermia. Hum Reprod. 26(11):2944-9. Epub 2011 Aug 19.

[25] Oliveira JB, Cavagna M, Petersen CG, Mauri AL, Massaro FC, Silva LF, Baruffi RL, Franco JG Jr. 2011. Pregnancy outcomes in women with repeated implantation failures after intracytoplasmic morphologically selected sperm injection (IMSI). Reprod Biol Endocrinol. 22;9:99.

[26] Keefe D, Liu L, Wang W, Silva C. 2003. Imaging meiotic spindles by polarization light microscopy; principles and applications to IVF. Reprod Biomed Online. 7(1):24-9. Review.

[27] Korkmaz C, Cinar O, Akyol M. 2011. The relationship between meiotic spindle imaging and outcome of intracytoplasmic sperm injection: a retrospective study. Gynecol Endocrinol. 27(10):737-41. Epub 2010 Sep 9.

[28] Coticchio G, Sciajno R, Hutt K, Bromfield J, Borini A, Albertini DF. 2010. Comparative analysis of the metaphase II spindle of human oocytes through polarized light and high-performance confocal microscopy. Fertil Steril. 93(6):2056-64. Epub 2009 Feb 24.

[29] De Vos A, Van de Velde H, Joris H, Van Steirteghem A. 1999. In-vitro matured metaphase-I oocytes have a lower fertilization rate but similar embryo quality as mature metaphase-II oocytes after intracytoplasmic sperm injection. Hum Reprod. 14:1859-1863.

[30] Strassburger D, Friedler S, Raziel A, Kasterstein E, Schachter M, Ron-El R. The outcome of ICSI of immature MI oocytes and rescued in vitro matured MII oocytes. Hum Reprod 2004;19:1587-1590.

[31] Eppig JJ, Schultz RM, O'Brien Mand Chesnel, M. 1994. Relationship between the developmental programs controlling nuclear and cytoplasmic maturation of mouse oocytes. Develop Biol. Vol 164 (1) 1-9.

[32] Sundstromand P, Nilsson BO. 1988. Meiotic and cytoplasmic maturation of oocytes collected in stimulated cycles is asynchronous. Hum Reprod Vol 3 (5) 613-619

[33] Balakier H, Sojecki A, Motamedi G, Librach C 2004. Time dependent capability of human oocytes for activation and pronuclear formation during metaphase II arrest," Hum Reprod. Vol 19(4) 982-987

[34] Falcone P, Gambera L, Pisoni M, Lofiego V, De Leo V, Mencaglia L, Piomboni P. 2008. Correlation between oocyte preincubation time and pregnancy rate after intracytoplasmic sperm injection. Gynecol Endocrinol. 24(6):295-9. 
[35] Rienzi L, Ubaldi F, Anniballo R, Cerulo G, Greco E. 1998. Preincubation of human oocytes may improve fertilization and embryo quality after intracytoplasmic sperm injection. Hum Reprod 13(4):1014-1019.

[36] Ho JY,Chen MJ,Yi YC, Guu HF, Ho ES. 2003. The effect of preincubation period of oocytes on nuclear maturity, fertilization rate, embryo quality, and pregnancy outcome in IVFandICSI1," J Assist Reprod Genet. Vol 20 (9) 358-364

[37] Isiklar A,Mercan R, Balaban B, Alatas C, Aksoy S, Urman B. 2004. Impact of oocyte preincubation time on fertilization, embryo quality and pregnancy rate after intracy toplasmic sperm injection. Reprod BioMed Online. 8 (6) 682-686

[38] Yanagida K, Yazawa H, Katayose H, Suzuki K, Hoshi K, Sato A. 1998. Influence of oocyte preincubation time on fertilization after intracytoplasmic sperm injection. Hum Reprod Vol 13 (8) 2223-2226.

[39] Malgorzata K, Depa-Martynów M, Butowska W et al. 2007 Human spermatozoa ultrastructure assessment in the infertility treatment by assisted reproduction technique. Arch Androl 53, 297-302.

[40] Francavilla S, Cordeschi G, Pelliccione F et al. 2007 Isolated teratozoospermia: a cause of male sterility in the era of ICSI? Front Biosci 12, 69-88.

[41] Kupker W, Schulze W, Diedrich K 1998 Ultrastructure of gametes and intracytoplasmic sperm injection. The significance of sperm morphology. Hum Reprod 13 (Suppl. 1), 99-106.

[42] Yu JJ, Xu YM 2004 Ultrastructural defects of acrosome in infertile men. Arch Androl 50, 405-409.

[43] Lamb DJ 1999 Debate: Is ICSI a Genetic Bomb? Yes. J Androl20, 23-33.

[44] Zhang S, Wang N, He B, Cheng J, Xi S, Wang SM, Gao Y, Wang J. 2012. Sperm head vacuoles-light microscopic and ultrastructural observations: a case report. Ultrastruct Pathol. 36(3):185-8.

[45] Perdrix A, Saïdi R, Ménard JF, Gruel E, Milazzo JP, Macé B, Rives N. 2012. Relationship between conventional sperm parameters and motile sperm organelle morphology examination (MSOME). Int J Androl. Mar 15. doi: 10.1111/j.1365-2605.2012.01249.x. [Epub ahead of print]

[46] Cassuto NG, Hazout A, Hammoud I, Balet R, Bouret D, Barak Y, Jellad S, Plouchart JM, Selva J, Yazbeck C. 2012. Correlation between DNA defect and sperm-head morphology. Reprod Biomed Online. 24(2):211-8. Epub 2011 Oct 22.

[47] Guthauser B, Albert M, Ferfouri F, Ray PF, Rabiey G, Selva J, Vialard F. 2011. Inverse correlation between chromatin condensation and sperm head size in a case of enlarged sperm heads. Reprod Biomed Online. 23(6):711-6. Epub 2011 Jul 22.

[48] Dam AH, Feenstra I, Westphal JR et al. 2007 Globozoospermia revisited. Hum Reprod Update 13, 63-75.

[49] Kullander S, Rausing A 1975. On round-headed human spermatozoa. International Journal of Fertility 20, 33-40.

[50] Kilani Z, Ismail R, Ghunaim S et al. 2004 Evaluation and treatment of familial globozoospermia in five brothers. Fertil steril 82, 1436-1439.

[51] Wan L, An LM, Xia XY. 2011. Molecular genetics of globozoospermia: an update. Zhonghua Nan Ke Xue. 17(10):935-8. Review. Chinese. 
[52] Battaglia DE, Koehler JK, Klein NA et al. 1997 Failure of oocyte activation after intracytoplasmic sperm injection using round-headed sperm. Fertil steril 68, 118-122.

[53] Rybouchkin AV, Van der Straeten F, Quatacker J et al. 1997 Fertilization and pregnancy after assisted oocyte activation and intracytoplasmic sperm injection in a case of roundheaded sperm associated with deficient oocyte activation capacity. Fertil steril 68, 11441147.

[54] Stone S, O'Mahony F, Khalaf Y et al. 2000 A normal live birth after intracytoplasmic sperm injection for globozoospermia without assisted oocyte activation. Hum Reprod 15, 139-141.

[55] Edirisinghe WR, Murch AR, Junk SM et al. 1998 Cytogenetic analysis of unfertilized oocytes following intracytoplasmic sperm injection using spermatozoa from a globozoospermic man. Hum Reprod 13, 3094-3098.

[56] Aitken RJ, De Iuliis GN 2007 Origins and consequences of DNA damage in male germ cells. Reprod BioMed Online 14, 727-733.

[57] Virro MR, Larson-Cook KL, Evenson DP 2004 Sperm chromatin structure assay (SCSA) parameters are related to fertilization, blastocyst development, and ongoing pregnancy in in vitro fertilization and intracytoplasmic sperm injection cycles. Fertil steril 81, 1289-1295.

[58] Braude P, Bolton V, Moore S 1988 Human gene expression first occurs between the four- and eight-cell stages of preimplantation development. Nature 332, 459-461.

[59] Twigg JP, Irvine DS, Aitken RJ 1998 Oxidative damage to DNA in human spermatozoa does not preclude pronucleus formation at intracytoplasmic sperm injection. Hum Reprod 13, 1864-1871.

[60] Tesarik J, Greco E, Mendoza C 2004 Late, but not early, paternal effect on human embryo development is related to sperm DNA fragmentation. Hum Reprod 19, 611-615.

[61] Sun JG, Jurisicova A, Casper RF 1997 Detection of deoxyribonucleic acid fragmentation in human sperm: correlation with fertilization in vitro. Biol Reprod 56, 602-607.

[62] Irvine DS, Twigg JP, Gordon EL et al. 2001 DNA integrity in human spermatozoa: relationships with semen quality. J Androl21, 33-44.

[63] Lopes S, Jurisicova A, Casper RF 1998 Gamete-specific DNA fragmentation in unfertilized human oocytes after intracytoplasmic sperm injection. Hum Reprod 13, 703-708.

[64] Sikora J, Kempisty B, Jedrzejczak P et al. 2006 Influence of DNA damage on fertilizing capacity of spermatozoa. Przeglad lekarski 63, 800-802.

[65] Moskovtsev S, Alladin N, Lo K, Jarvi K, Mullen J, Librach C. 2012. A comparison of ejaculated and testicular spermatozoa aneuploidy rates in patients with high sperm DNA damage. Syst Biol Reprod Med. 2012 Mar 20. [Epub ahead of print]

[66] Evenson DP, Larson KL, Jost LK 2002 Sperm chromatin structure assay: its clinical use for detecting sperm DNA fragmentation in male infertility and comparisons with other techniques. J Androl23, 25-43.

[67] Potts RJ, Newbury CJ, Smith G et al. 1999 Sperm chromatin changes associated with male smoking. Mutation Research 423, 103-11.

[68] Bungum M, Humaidan P, Axmon A et al. 2007 Sperm DNA integrity assessment in prediction of assisted reproduction technology outcome. Hum Reprod 22, 174-179. 
[69] Spano M, Seli E, Bizzaro D et al. 2005 The significance of sperm nuclear DNA strand breaks on reproductive outcome. Current Opinion in Obstetrics \& Gynecology 17, 255-260.

[70] Aitken RJ, De Iuliis GN, McLachlan RI 2009 Biological and clinical significance of DNA damage in the male germ line. Int J Androl 32, 46-56.

[71] Beshay VE, Bukulmez O. 2012. Sperm DNA damage: how relevant is it clinically? Curr Opin Obstet Gynecol 24(3):172-9.

[72] Saremi A, Esfandiari N, Salehi N et al. 2002 The first successful pregnancy following injection of testicular round spermatid in Iran. Archiv Androl48, 315-319.

[73] Jurisicova A, Lopes S, Meriano J et al. 1999 DNA damage in round spermatids of mice with targeted disruption of PP1c gamma gene and in testicular biopsies of patients with non-destructive azoospermia. Mol Hum Reprod 5, 323-330.

[74] Zech H, Vanderzwalmen P, Prapas Y et al. 2000 Congenital malformations after intracytoplasmic injection of spermatids. Hum Reprod 15, 969 -971.

[75] The Practice Committee of American Society for Reproductive Medicine, Practice Committee of Society for Assisted Reproductive Technology 2008 Round spermatid nucleus injection (ROSNI). Fertil steril 90 (Supp 1), S199-201.

[76] Johnson RT, Rao PN 1970 Mammalian cell fusion: induction of premature chromosome condensation in interphase nuclei. Nature 226, 717-722.

[77] Schmiady H, Sperling K, Kentenich H et al. 1986 Prematurely condensed human sperm chromosomes after in vitro fertilization (IVF). Human Genetics 74, 441-443.

[78] Ma S, Kalousek DK, Yuen BH 1994 Chromosome investigation in in vitro fertilization failure. J Assist Reprod Gen1,445-451.

[79] Van Blerkom J, Davis PW, Merriam J 1994 Fertilization and early embryology: a retrospective analysis of unfertilized and presumed parthenogenetically activated human oocytes demonstrates a high frequency of sperm penetration. Hum Reprod 9, 2381-2388.

[80] Yanagida K, Katayose H, Yazawa H et al. 1999 Successful fertilization and pregnancy following ICSI and electrical oocyte activation. Hum Reprod 14, 1307-1311.

[81] Grasa P, Coward K, Young C et al. 2008 The pattern of localization of the putative oocyte activation factor, phospholipase $\mathrm{Cz}$, in uncapacitated, capacitated, and ionophore-treated human spermatozoa. Hum Reprod 23, 2513-2522.

[82] Balhorn R 1982. A model for the structure of chromatin in mammalian sperm. The Journal of Cell Biology 93, 298-305.

[83] Dozortsev D, Qian C, Ermilov A et al. 1997 Sperm-associated oocyte-activating factor is released from the spermatozoon within 30 minutes after injection as a result of the sperm-oocyte interaction. Hum Reprod 12, 2792-2796.

[84] Ma S, Yuen BH 2001 Intracytoplasmic sperm injection could minimize the incidence of prematurely condensed human sperm chromosomes. Fertil Steril 75, 1095-1101.

[85] Calafell JM, Badenas J, Egozcue J et al. 1991 Premature chromosome condensation as a sign of oocyte immaturity. Hum Reprod 6, 1017-1021.

[86] Rosenbuch BE 2000 Frequency and patterns of premature sperm chromosome condensation in oocytes failing to fertilize after intracytoplasmic sperm injection. J Assist Reprod Gen17, 253-259. 
[87] Schmiady H, Schulze W, Scheiber I et al. 2005 High rate of premature chromosome condensation in human oocytes following microinjection with round-headed sperm: Case report. Hum Reprod 20, 1319-1323.

[88] Nasr-Esfahani MH, Razavi S, Tavalaee M 2008 Failed fertilization after ICSI and spermiogenic defects. Fertil Steril 89, 892-898.

[89] Chemes H, Alvarez Sedo C. 2012. Tales of the tail and sperm head aches: changing concepts on the prognostic significance of sperm pathologies affecting the head, neck and tail. Asian J Androl. 14(1):14-23. doi: 10.1038/aja.2011.168. Epub 2011 Dec 26.

[90] Konc J, Kanyo K, Cseh S 2006 Deliveries from embryos fertilized with spermatozoa obtained from cryopreserved testicular tissue. J Assist Reprod Gen23, 247-252.

[91] Wang CW, Lai YM, Wang ML et al. 1997 Pregnancy after intracytoplasmic injection of immotile sperm. A case report. J Reprod Med42, 448-450.

[92] Kusakabe H, Szczygiel MA, Whittingham DG et al. 2001 Maintenance of genetic integrity in frozen and freeze-dried mouse spermatozoa. Proceedings of the National Academy of Sciences of the United States of America 98, 13501-13506.

[93] Ghosh S, Chattopadhyay R, Bose G, Ganesh A, Das S, Chakravarty BN. 2012. Selection of birefringent spermatozoa under Polscope: effect on intracytoplasmic sperm injection outcome. Andrologia. Feb 28. doi: 10.1111/j.1439-0272.2011.01258.x. [Epub ahead of print]

[94] Sallam H, Farrag A, Agameya A, El-Garem Y, Ezzeldin F. 2005. The use of the modified hypo-osmotic swelling test for the selection of immotile testicular spermatozoa in patients treated with ICSI: a randomized controlled study. Hum Reprod 2005; 20: 3435-40.

[95] Gerber P, Kruse R, Hirchenhain J, Kru“ ssel J, Neumann N. 2008. Pregnancy after laser assisted selection of viable spermatozoa before intracytoplasmatic sperm injection in a couple with male primary cilia dyskinesia. Fertil Steril 2008; 89: 1826.e9-12.

[96] Kordus R, Price R, Davis J, Whitman-Elia G. Successful twin birth following blastocyst culture of embryos derived from the immotile ejaculated spermatozoa from a patient with primary ciliary dyskinesia: a case report. J Assist Reprod Genet 2008; 25: 437-43.

[97] Mangoli V, Mangoli R, Dandekar S, Suri K, Desai S. Selection of viable spermatozoa from testicular biopsies: a comparative study between pentoxifylline and hypoosmotic swelling test. Fertil Steril 2011; 95: 631-4.

[98] Hattori H, Nakajo Y, Ito C, Toyama Y, Toshimori K, Kyono K. 2011. Birth of a healthy infant after intracytoplasmic sperm injection using pentoxifylline-activated sperm from a patient with Kartagener's syndrome. Fertil Steril. 95(7):2431.e9-11. Epub 2011 Apr 20.

[99] Jeyendran RS, Van der Ven HH, Perez-Pelaez M et al. 1984 Development of an assay to assess the functional integrity of the human sperm membrane and its relationship to other semen characteristics. J Reprod Fertil 70, 219-228.

[100] Casper RF, Meriano JS, Jarvi KA et al. 1996 The hypo-osmotic swelling test for selection of viable sperm for intracytoplasmic sperm injection in men with complete asthenozoospermia. Fertil Steril 65, 972-976.

[101] Liu V, Tsai YL, Katz E et al. 1997 High fertilization rate obtained after intracytoplasmic sperm injection with $100 \%$ nonmotile spermatozoa selected by using a simple modified hypo-osmotic swelling test. Fertil Steril 68, 373-375. 
[102] Ahmadi A, Ng SC 1997 The single sperm curling test, a modified hypo-osmotic swelling test, as a potential technique for the selection of viable sperm for intracytoplasmic sperm injection. Fertil Steril 68, 346-350.

[103] Verheyen G, Joris H, Crits K et al. 1997 Comparison of different hypo-osmotic swelling solutions to select viable immotile spermatozoa for potential use in intracytoplasmic sperm injection. Hum Reprod Update 3, 195-203.

[104] Sallam HN, Farrag A, Agameya AF et al. 2001 The use of a modified hypo-osmotic swelling test for the selection of viable ejaculated and testicular immotile spermatozoa in ICSI. Hum Reprod 16, 272-276.

[105] Yildirim G, Ficicioglu C, Akcin O, Attar R, Tecellioglu N, Yencilek F (2009). Can pentoxifylline improve the sperm motion and ICSI success in the primary ciliary dyskinesia? Arch Gynecol Obstet. 279(2):213-5. Epub 2008 May 7.

[106] Palermo GD, Joris H, Devroey P et al. 1992 Pregnancies after intracytoplasmic injection of single spermatozoon into an oocyte. Lancet 340, 17-18.

[107] Proctor M, Johnson N, Van Peperstraten AM et al. 2009 Techniques for surgical retrieval of sperm prior to intra-cytoplasmic sperm injection (ICSI) for azoospermia. Cochrane Database for Systematic Reviews 2, CD002807.

[108] Wennerholm UB, Bergh C, Hamberger L et al. 2000 Obstetric outcome of pregnancies following ICSI, classified according to sperm origin and quality. Hum Reprod 15, 11891194.

[109] Bulkumez O, Yucel A, Yarali H et al. 2001 The origin of spermatozoa does not affect intracytoplasmic sperm injection outcome. Eur J Obstet Gyn Reprod Biol94, 250-255.

[110] Silber SJ, Nagy ZP, Liu J 1994 Conventional in-vitro fertilization vs ICSI for patients requiring microsurgical sperm aspiration. Hum Reprod 9, 1705-1709.

[111] Lewis S, Klonoff-Cohen H 2005 What factors affect intracytoplasmic sperm injection outcomes? Obstet \& Gynecol Sur 60, 111-123.

[112] Matyas S, Papp G, Kovacs P et al. 2005 Intracytoplasmic injection with motile and immotile frozen-thawed testicular spermatozoa (the Hungarian experience). Andrologia 37, 25-28.

[113] Halliday J. 2012. Outcomes for offspring of men having ICSI for male factor infertility. Asian J Androl. 14(1):116-20. doi: 10.1038/aja.2011.71. Epub 2011 Dec 12.

[114] Yamano S, Nakagawa K, Nakasaka H et al. 2000 Fertility failure and oocyte activation. J Med Invest 47, 1-8.

[115] Miyazaki S, Ito M 2006 Calcium signals for egg activation in mammals. J Pharmacol Sci 100, 545-552.

[116] Swann K 1990 A cytosolic sperm factor stimulates repetitive calcium increases and mimics fertilization in hamster eggs. Development 110, 1295-1302.

[117] Wu H, He CL, Fissore RA 1997 Injection of a porcine sperm factor triggers calcium oscillations in mouse oocytes and bovine eggs. Mol Reprod Develop 46, 176-189.

[118] Jones KT, Cruttwell C, Parrington J, Swann K 1998 A mammalian sperm cytosolic phospholipase $\mathrm{C}$ activity generates inositol trisphosphate and causes $\mathrm{Ca} 2+$ release in sea urchin egg homogenates. FEBS Letters 437, 297-300. 
[119] Wu H, Smyth J, Luzzi V et al. 2001. Sperm factor induces intracellular free calcium oscillations by stimulating the phosphoinositide pathway. Biol Reprod 64, 1338-1349.

[120] Rice A, Parrington J, Jones KT et al. 2000 Mammalian sperm contain a Ca(2+)-sensitive phospholipase $\mathrm{C}$ activity that can generate InsP3 from PIP2 associated with intracellular organelles. Develop Biol 228, 125-135.

[121] Saunders CM 2002 PLC : a sperm-specific trigger of $\mathrm{Ca}(2+)$ oscillations in eggs and embryo development. Development 129, 3533-3544.

[122] Kouchi Z, Fukami K, Shikano T et al. 2004 Recombinant phospholipase Czeta has high $\mathrm{Ca} 2+$ sensitivity and induces Ca2+ oscillations in mouse eggs. J Biol Chem 279, 1040810412.

[123] Kurokawa M, Yoon SY, Alfandari D et al. 2007 Proteolytic processing of phospholipase Czeta and [Ca2+]i oscillations during mammalian fertilization. Develop Biol 312, 407-418.

[124] Yoon SY, Jellerette T, Salicioni AMJ et al. 2008 Human sperm devoid of PLC, zeta 1 fail to induce $\mathrm{Ca}(2+)$ release and are unable to initiate the first step of embryo development. The Journal of Clinical Investigation 118, 3671-3681.

[125] Heindryckx B, Van der Elst J, De Sutter P et al. 2005 Treatment option for sperm- or oocyte-related fertilization failure: assisted oocyte activation following diagnostic heterologous ICSI. Hum Reprod 20, 2237-2241.

[126] Vanden MF, Nikiforaki D, De Gheselle S, Dullaerts V, Van den Abbeel E, Gerris J, Heindryckx B, De Sutter P. Assisted oocyte activation is not beneficial for all patients with a suspected oocyte-related activation deficiency. Hum Reprod. 2012 Apr 4. [Epub ahead of print]

[127] Nakada K, Mizuno J 1998 Intracellular calcium responses in bovine oocytes induced by spermatozoa and by reagents. Theriogenology 50, 269-282.

[128] Cuthbertson KSR, Whittingham DG, Cobbold PH 1981. Free Ca2+ increases in exponential phases during mouse oocyte activation. Nature 294, 754-757.

[129] Loi P, Ledda S, Fulka Jr J et al. 1998 Development of parthenogenetic and cloned ovine embryos: effect of activation protocols. Biol Reprod 58, 1177-1187.

[130] Hoshi K, Yanagida K, Yazawa H et al. 1995 Intracytoplasmic sperm injection using immobilized or motile human spermatozoon. Fertil Steril 63, 1241-1245.

[131] Lu Q, Zhao Y, Gao X et al. 2006 Combination of calcium ionophore A23187 with puromycin salvages human unfertilized oocytes after ICSI. Eur J Obstet Gyn Reprod Biol126, 72-76.

[132] Kyono K, Kumagai S, Nishinaka C et al. 2008 Birth and follow-up of babies born following ICSI using $\mathrm{SrCl}_{2}$ oocyte activation. Reprod BioMed Online 17, 53-58.

[133] Yanagida K, Morozumi K, Katayose H et al. 2006 Case report: Successful pregnancy after ICSI with strontium oocyte activation in low rates of fertilization. Reprod BioMed Online 3, 801-806.

[134] Ozil JP 1990 The parthenogenetic development of rabbit oocytes after repetitive pulsatile electrical stimulation. Development 109, 117-127.

[135] Kimura Y, Yanagimachi R 1995 Development of normal mice from oocytes injected with secondary spermatocyte nuclei. Biol Reprod 53, 855-862. 
[136] Zhang J, Wang C, Blaszcyzk A et al. 1999 Electrical activation and in vitro development of human oocytes that fail to fertilize after intracytoplasmic sperm injection. Fertil Steril 72, 509-512.

[137] Manipalviratn S, Ahnonkitpanit V, Numchaisrika P et al. 2006 Results of direct current electrical activation of failed-to-fertilize oocytes after intracytoplasmic sperm injection. J Reprod Med51, 493-499.

[138] Saldeen P, Källen K, Sundström P 2007 The probability of successful IVF outcome after poor ovarian response. Acta Obstet Gynecol Scan 86, 457-461.

[139] Kailasam C, Keay SD, Wilson P et al. 2004 Defining poor ovarian response during IVF cycles, in women $<40$ years, and its relationship with treatment outcome. Hum Reprod 19, 1544-1547.

[140] Sun W, Stegmann BJ, Henne M et al. 2008 A new approach to ovarian reserve testing. Fertility Sterility 90, 2196-2202.

[141] Lashen H, Ledger W, Lopez-Bernal A et al. 1999 Poor responders to ovulation induction-is proceeding to in-vitro fertilization worthwhile? Hum Reprod 14, 964- 969.

[142] Yanagida K 2004 Complete fertilization failure in ICSI. Human Cell 17, 187-193.

[143] Melie NA, Adeniyi OA, Igbineweka OM et al. 2003 Predictive value of the number of oocytes retrieved at ultrasound-directed follicular aspiration with regard to fertilization rates and pregnancy outcome in intracytoplasmic sperm injection treatment cycles. Fertil Steril 80, 1376-1379.

[144] Devroey P, Godoy H, Smitz J et al. 1996 Female age predicts embryonic implantation after ICSI: a case-controlled study. Hum Reprod 11, 1324-1327.

[145] Tietze C 1957 Reproductive span and rate of reproduction among Hutterite women. Fertil Steril 8, 89-97.

[146] Baker TG 1963 A Quantitative and Cytological Study of Germ Cells in Human Ovaries. Proceedings of the Royal Society of London, Series B, Biological Sciences 158, 417-433.

[147] Djahanbakhch O, Ezzati M, Zosmer A 2007 Reproductive ageing in women. The Journal of Pathology 211, 219-231.

[148] Shu Y, Gebhardt J, Watt J et al. 2007 Fertilization, embryo development, and clinical outcome of immature oocytes from stimulated intracytoplasmic sperm injection cycles. Fertil Steril 87, 1022-1027.

[149] Smitz J, Cortvrindt R 1999 Oocyte in-vitro maturation and follicle culture: current clinical achievements and future directions. Hum Reprod 14, 145-161.

[150] Chen SU, Chen HF, Lien YR et al. 2000 Schedule to inject IVM-MI oocytes may increase pregnancy after intracytoplasmic sperm injection. Archiv Androl 44, 197-205.

[151] Sun QY, Lai L, Bonk A et al. 2001 Cytoplasmic changes in relation to nuclear maturation and early embryo developmental potential of porcine oocytes: effects of gonadotropins, cumulus cells, follicular size, and protein synthesis inhibition. Molecular Reprod Develop 59, 192- 198.

[152] Xia P 1997 Intracytoplasmic sperm injection: correlation of oocyte grade based on polar body, perivitelline space and cytoplasmic inclusions with fertilization rate and embryo quality. Hum Reprod 12, 1750-1755. 
[153] Van Blerkom J, Henry G 1992 Oocyte dysmorphism and aneuploidy in meiotically mature oocytes after ovarian stimulation. Hum Reprod 7, 379-390.

[154] Ebner T, Moser M, Tews G 2006 Is oocyte morphology prognostic of embryo developmental potential after ICSI? Reprod BioMed Online 12, 507-512.

[155] Meriano JS, Alexis J, Visram-Zaver S et al. 2001 Tracking of oocyte dysmorphisms for ICSI patients may prove relevant to the outcome in subsequent patient cycles. Hum Reprod 16, 2118-2123.

[156] Mikkelsen AL, Lindenberg S 2001 Morphology of in-vitro matured oocytes: impact on fertility potential and embryo quality. Hum Reprod 16, 17I4-I7I8.

[157] Ciotti PM, Nmarangelo L, Morsclli-Labate AM et al. 2004 First polar body morphology before ICSI is not related to embryo quality or pregnancy rate. Hum Reprod 19, 23342339.

[158] Otsuki J, Okada A, Morimoto K et al. 2004 The relationship between pregnancy outcome and smooth endoplasmic reticulum clusters in metaphase II human oocytes. Hum Reprod 19, 1591- 1597.

[159] De Santis L, Cino I, Rabellotti E et al. 2005 Polar body morphology and spindle imaging as predictors of oocyte quality. Reprod BioMed Online 11, 36-42.

[160] Ten J, Mendiola J, Vioque J et al. 2007 Donor oocyte dysmorphisms and their influence on fertilization and embryo quality. Reprod BioMed Online 14, 40-48.

[161] Esfandiari N, Burjaq H, Gotlieb L et al. 2006. Brown oocytes: implications for assisted reproductive technology. Fertil Steril 86, 1522-1525.

[162] Serhal PF, Ranieri DM, Kinis A et al. 1997 Oocyte morphology predicts outcome of intracytoplasmic sperm injection. Hum Reprod 12, 1267-1270.

[163] Katz N, Tur-Kaspa I 2000 Cytoplasmic maturity of metaphase II human oocytes: Biologic importance and clinical implications for in vitro fertilization. Reprod Technol 10, 170-173.

[164] Esfandiari N, Ryan EA, Gotlieb L et al. 2005 Successful pregnancy following transfer of embryos from oocytes with abnormal zona pellucida and cytoplasm morphology. Reprod BioMed Online 11, 620-623.

[165] JelinkovaL, Pavelkova J, Rezabek K et al. 2001 Treatment of infertility using oocytes without the zona pellucida. Ceská gynekologie 65, 456-459.

[166] Stanger JD, Stevenson K, Lakmaker A et al. 2001 Pregnancy following fertilization of zona-free, coronal cell intact human ova: Case Report. Hum Reprod 16, 164-167.

[167] Naito K, Toyoda Y, Yanagimachi R 1992 Production of normal mice from oocytes fertilized and developed without zonae pellucidae. Hum Reprod 7, 281-285.

[168] Wu GM, Lai L, Mao J et al. 2004 Birth of piglets by in vitro fertilization of zona-free porcine oocytes. Theriogenology 62, 1544-1556.

[169] Moomjy M, Sills ES, Rosenwaks Z et al. 1998 Implications of complete fertilization failure after intracytoplasmic sperm injection for subsequent fertilization and reproductive outcome. Hum Reprod 13, 2212-2216.

[170] Rouzi AA, Amarin Z 2002 Repeat intracytoplasmic sperm injection. Clinical perspective. Saudi Med J 23, 1470-1472.

[171] Wen SW, Walker M, Léveillé MC et al. 2004 Intracytoplasmic sperm injection: promises and challenges. Can Med Ass J 171, 845-846. 